[16] J. E. Ford et M. E. GRegory. Rep. Nat. Inst. Dairy, p. 109. 1957.

[17] A. Frangois et J. Hermier. Résumés des Communioations. I Ve Congrès International de Nutrition. Paris, p. 147. 1957.

\title{
DÉTERMINATION DE LA DURÉE DE CHAUFFAGE DANS UN APPAREIL DE STÉRILISATION DU LAIT A TRÈS HAUTE TEMPÉRATURE
}

par

\section{J. HERMIER, J. VERGE et G. GROSCLAUDE}

Station centrale de Microbiologie et Recherches laitières Jouy-en-Josas (Seine-et-Oise) France

\section{I. - INTRODUCTION}

Dans les procédés de fabrication du lait stérilisé actuellement en usage, le lait est, avant la mise en bouteilles, chauffé dans un "préstérilisateur " à $130-145^{\circ} \mathrm{C}$., pendant 1 à 20 secondes, puis refroidi à la température d'embouteillage $\left(65-70^{\circ} \mathrm{C}\right.$.). Puisque cette opération, dite "préstérilisation », a pour but de détruire les spores que pouvait contenir le lait cru, il parait nécessaire de connaître les caractéristiques de l'action sporicide du préstérilisateur. Celles-ci ne peuvent être évaluées que si l'on connaît les caractéristiques du traitement thermique : durée du traitement, évolution de la température pendant le traitement, distribution des vitesses d'écoulement.

$\mathrm{Au}$ point de vue du traitement thermique, les préstérilisateurs (ou appareils de stérilisation à très haute température) peuvent se classer en deux catégories :

- Préstéritisateurs à chauffage indirect (type A.P.V., Stork), où le lait est séparé du fluide chauffant (vapeur) ou refroidissant (eau froide) par une paroi métallique. La montée en température du lait est d'environ 20 secondes, le chambrage de 2 à 16 secondes, la descente en température d'environ 20 secondes. Il faut donc tenir compte de l'effet léthal de la chaleur non seulement pendant le chambrage, mais aussi pendant la montée et la descente en température. Ce problème complexe vient d'être traité par Burton [1].

- Préstérilisateurs à chauffage direct (type Upérisateur et appareil Laguilharre) : le lait est en contact avec la vapeur dans la section de chauffage ; l'eau condensée pendant le chauffage est séparée du lait par détente sous vide du mélange eau-lait dans la section de refroidissement. La montée et la descente en température sont instantanées et l'effet léthal de la chaleur ne se produit que pendant le chauffage. Il suffit donc de connaître la durée du chauffage 
et la répartition des vitesses dans la section de chauffage pour pouvoir calculer l'effet sporicide du préstérilisateur.

Le but du présent travail est : $a$ ) de déterminer la durée du chauffage dans un préstérilisateur du type Laguilharre ; b) de vérifier les résultats obtenus par l'étude de la destruction, par le préstérilisateur, des spores d'une souche de Bacillus subtilis.

\section{II. - DESCRIPTION DU STÉRILISATEUR}

Les mesures ont été effectuées sur un stérilisateur à très haute température qui est un modèle réduit, adapté aux besoins du laboratoire, du préstérilisateur industriel Laguilharre (1) et qui a déjà été utilisé dans un travail précédent [3].

Principe. - Le lait est finement pulvérisé à l'intérieur d'une enceinte (caisse de stérilisation) contenant de la vapeur saturante sous pression. La division en fines goutelettes entraîne une grande surface de condensation de la vapeur et permet ainsi au lait d'atteindre instantanément la température de la vapeur. Après avoir traversé cette enceinte, le lait passe dans une seconde enceinte (caisse de détente) maintenue sous vide : la température du lait s'abaisse brusquement et le lait abandonne une certaine quantité d'eau sous forme de vapeur ; cette quantité d'eau correspond à la quantité de vapeur condensée lors du séjour dans la première enceinte, quand la température du lait à l'entrée de la caisse de stérilisation et celle dans la caisse de détente sont égales.

Circuit du lait (fig. 1 et 2). - Le lait à traiter, qui est contenu dans un bac d'alimentation cylindrique $\left(B_{1}\right)$ de 50 litres de capacité, est injecté par une pompe volumétrique (PI) dans la caisse de stérilisation; un échangeur tubulaire $\left(\mathrm{E}_{1}\right)$, chauffé à la vapeur, est intercalé entre la pompe d'injection et la caisse de stérilisation; il permet, si on le désire, de porter le lait avant son entrée dans la caisse de stérilisation à une température comprise entre 30 et $90^{\circ} \mathrm{C}$. La caisse de stérilisation $\left(\mathrm{C}_{1}\right)$ est constituée par un cylindre de $0 \mathrm{~m} .25$ de diamètre et de $0 \mathrm{~m}$. 80 de hauteur, fermé par un couvercle démontable; ce dernier est muni d'un robinet qui doit rester légèrement ouvert pour permettre l'élimination des gaz incondensables. Le lait, pulvérisé par une buse à la partie supérieure de la caisse de stérilisation, est recueilli dans le bas de la caisse et passe dans une caisse de détente $\left(\mathrm{C}_{2}\right)$ identique à la caisse de stérilisation; il est enfin recueilli dans un bac de réception $\left(\mathrm{B}_{2}\right)$ identique au bac d'alimentation, mais muni à la base d'un robinet de prélèvement et à la partie supérieure d'un robinet d'admission d'air comprimé stérile. La caisse de détente et le bac de réception sont maintenus sous vide par une pompe à anneau d'eau (PV);

(1) Appareil eonstruit par les Etablissements Laguilharre, Courbevoie (Seine) 


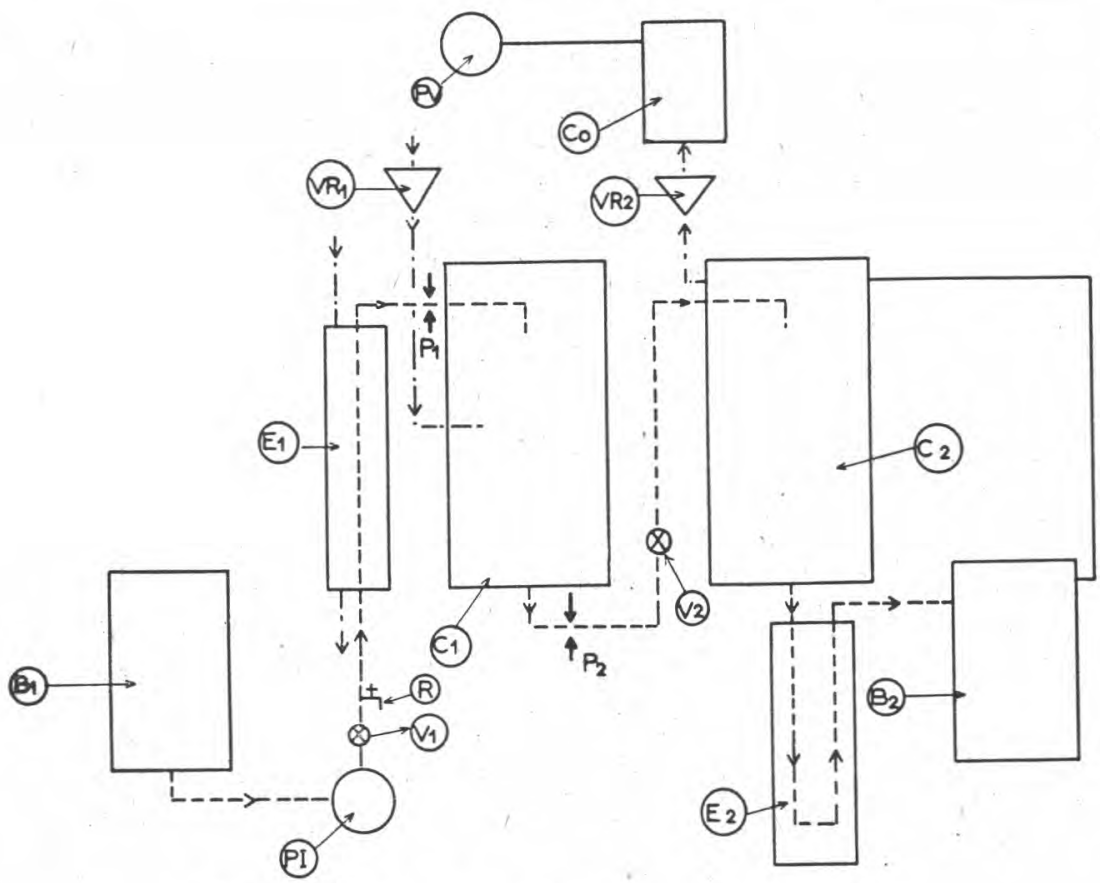

Fig. 1. - Schéma du stérilisateur de laboratoire.

$\left(B_{1}\right)$ Bac d'alimentation ; $\left(B_{2}\right)$ Bac de réception ; $\left(C_{1}\right)$ Caisse de stérilisation ; $\left(C_{2}\right)$ Caisse de détente; $(C o)$ Condenseur ; $\left(E_{1}\right)$ et $\left(E_{2}\right)$ Echangeurs ; $\left(P_{1}\right)$ et $\left(\mathrm{P}_{2}\right)$ Points de mesure : emplacement des cellules ; (PI) Pompe d'injection ; (PV) Pompe à vide; (R) Robinet d'injection de la solution de colorant; $\left(V_{1}\right)$ et $\left(V_{2}\right)$ Vannes de débit du lait; $\left(V_{R_{1}}\right)$ et $\left(V_{R_{2}}\right)$ Vannes régulatrices de pression de vapeur

- - - Circuit du lait.

-.... Circuit de la vapeur.

la vapeur libérée par le lait dans la caisse de détente est condensée dans un condenseur (Co) relié à la pompe à vide. Comme la température du lait à l'intérieur de la caisse de détente ne peut être inférieure à $35-40^{\circ} \mathrm{C}$., on peut intercaler, si on le désire, entre la caisse et le bac de réception un échangeur de température $\left(E_{2}\right)$ fonctionnant à l'eau ou à la saumure. Toutes les parties métalliques de l'appareil en contact avec le lait sont en acier inoxydable.

Deux vannes, $V_{1}$ et $V_{2}$, commandent la vitesse d'écoulement du lait ; la vanne $V_{1}$ commande le débit à la sortie de la pompe d'injection, donc le débit du stérilisateur; la vanne $V_{2}$ située entre les deux caisses doit être réglée de façon à éviter l'accumulation du lait dans la partie inférieure de la caisse de stérilisation.

Alimentation en vapeur. - La vapeur est fournie sous une 
Fig. 2. - Vue d'ensemble du stérilisateur.

On aperçoit, en haut et en avant de la crisse de stérilisation, la gaine de la pre. mière cellule photoélectrique (point de mesure $P_{1}$ ) et en bas et en arrière de la même caisse, la gaine de la seconde cellule (point de mesure $P_{2}$ ) en partie masquée.

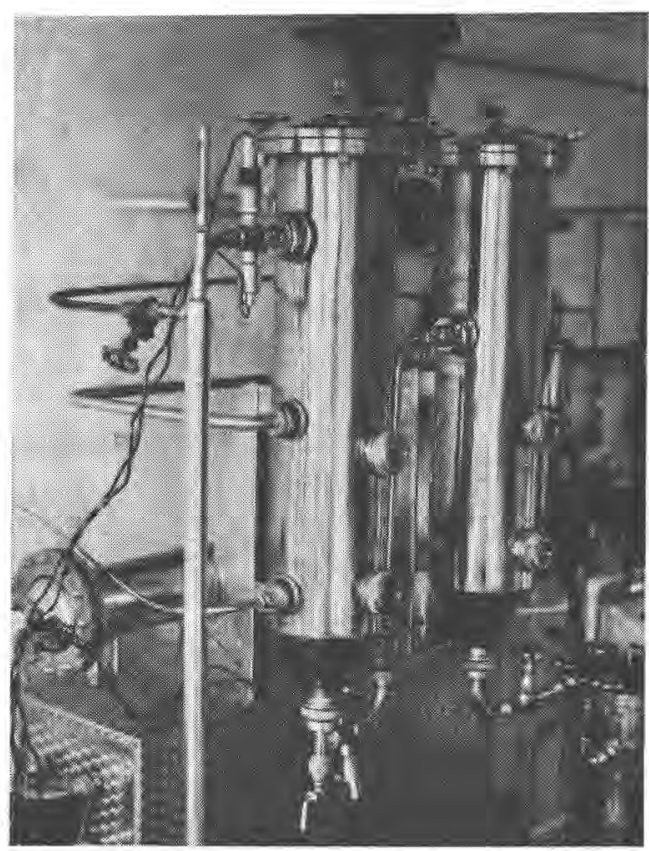

pression de 8 kilogrammes par un générateur de vapeur électrique Damond (25 KW, $65 \mathrm{~A})$. Une vanne $\left(\mathrm{VR}_{1}\right)$ commandée par un régulateur de pression, admet la quantité de vapeur nécessaire dans la caisse de stérilisation de façon à y maintenir une pression constante. Le régulateur est du type régulateur à membrane utilisant l'eau comme fluide auxiliaire : il permet une réponse instantanée aux variations de pression à l'intérieur de la caisse de stérilisation. Une vanne $\left(V_{R_{2}}\right)$ commandée par un système de régulation identique maintient une pression de vide constante dans la caisse de détente.

Puisque dans chacune des deux caisses, la pression est constante, il s'ensuit que la température y est également constante.

Débit de l'appareil. - Le débit de l'appareil est fonction du débit de la pompe commandé par la vanne $V_{1}$ et de la pression à l'intérieur de la eaisse de stérilisation done de la température de chauffage. Le débit maximum est fixé par la chaudière et diminue donc avec la température de traitement (voir tableau I).

Prélèvement du lait traité. - Après traitement de la quantité voulue de lait, le bac de réception est isolé du reste de l'appareil par la fermeture de deux vannes. Grâce à l'admission d'air comprimé stérilisé par passage à travers un tube-filtre rempli de coton stérile, 
le bac est mis en légère surpression pour permettre une sortie rapide du lait par le robinet de prélèvement.

\section{TABLEAU I}

$\begin{array}{ccccccc}\begin{array}{c}\text { No de } \\ \text { l'essai }\end{array} & \begin{array}{c}\text { Température } \\ \text { Degré C. }\end{array} & \begin{array}{c}\text { Pression } \\ \mathrm{kg} / \mathrm{cm}^{2}\end{array} & \begin{array}{c}\text { Position } \\ \text { Vanne I (1) }\end{array} & \begin{array}{c}\text { Position } \\ \text { Vanne II (1) }\end{array} & \begin{array}{c}\text { Débit } \\ \text { l/h }\end{array} & \begin{array}{c}\text { Temps de passagé } \\ \text { (en secondes) }\end{array} \\ 1 & 120 & 1,20 & 34 & 3 / 4 & 98 & 5 \\ 2 & 130 & 2,05 & 36 & 3 / 4 & 15 & 13,25 \\ 3 & 130 & 2,05 & 35 & 3 / 4 & 61 & 5,75 \\ 4 & 130 & 2,05 & 34 & 3 / 4 & 94 & 4,75 \\ 5 & 130 & 2,05 & 33 & 3 / 4 & 128(2) & 4,75 \\ 6 & 130 & 2,05 & 34 & 1 / 2 & 92 & 6 \\ 7 & 130 & 2,05 & 34 & 1 / 4 & 94 & 6 \\ 8 & 130 & 2,05 & 34 & 1 / 2 & 96 & 4,75 \\ 9 & 140 & 3,00 & 34 & 3 / 4 & 77(2) & 5,25 \\ 10 & 150 & 4,30 & 34 & 3 / 4 & 38(2) & 10\end{array}$

\section{III. - MESURE DE LA DURÉE DE CHAUfFAGE}

\section{A. - Choix d'une méthode de mesure}

Au moment où ont été entrepris ces essais, nous ne disposions que des données concernant la mesure du temps de chambrage dans les pasteurisateurs à pasteurisation haute. DAvis [2] a résumé les différentes méthodes utilisables qui peuvent se classer en deux catégories :

1) On injecte à l'entrée de la section de chambrage du pasteurisateur en fonctionnement une substance identifiable par une méthode physique ou chimique ; à la sortie de l'élément de cham. brage on prélève toutes les secondes, un échantillon dans lequel on recherche la substance injectée.

2) On dispose à l'entrée et à la sortie de l'élément de chambrage, deux points de mesure. L'appareil étant en fonctionnement, on injecte en amont du premier point de mesure une substance dont le passage au niveau de chaque point de mesure est enregistré sur un enregistreur à deux voies relié aux points de mesure. Trois techniques sont utilisables :

a) Points de mesure: sondes thermométriques. Produit injecté : lait chaud;

b) Points de mesure: électrodes. Produit injecté : chlorure de sodium ;

c) Points de mesure: cellules photoélectriques. Produit injecté : bleu de méthylène. Dans ce dernier cas, le pasteurisateur fonctionne avec de l'eau et non avec du lait.

(1) Graduation arbitraire.

(2) Débit maximum. 
La faible capacité de traitement du stérilisateur interdit l'emploi du premier groupe de méthodes de mesure : en effet, il est indispensable que le prélèvement des échantillons ne vienne pas troubler l'écoulement du liquide au niveau du point de prélèvement. Ceci n'est réalisable que si le volume de l'échantillon est suffisamment faible par rapport au volume du liquide passant dans le tuyau pendant le prélèvement. Tel n'étant pas le cas avec l'appareil dont nous disposons, on a décidé de s'orienter vers la méthode des points de mesure.

Des mesures de température préliminaires ayant montré que la température baissait rapidement après la vanne $V_{2}$, il n'a été prévu que deux points de mesure : $\mathrm{P}_{1}$ à l'entrée de la caisse de stérilisation, $\mathrm{P}_{2}$ entre la sortie de la caisse de stérilisation et la vanne $\mathrm{V}_{2}$.

La température à l'intérieur de la caisse de stérilisation est constante, quelle que soit la température du lait à l'entrée de la caisse : la méthode d'injection du lait chaud n'est donc pas utilisable. La méthode d'injection de chlorure de sodium a dû être également abandonnée : des essais préliminaires ont en effet montré que les variations du zéro (c'est-à-dire pendant le passage de l'eau sans chlorure de sodium) masquent presque complètement les déviations enregistrées au moment du passage de la solution de chlorure de sodium. Ceci s'explique par le fait qu'au niveau des points de mesure, le régime d'écoulement est un régime turbulent et que le niveau du liquide en $P_{1}$ est très variable.

L'effort principal a donc porté sur l'enregistrement du passage d'une solution de bleu de méthylène par des cellules photoélectriques. Ces dernières doivent être choisies avec soin, car les points $P_{1}$ et $P_{2}$ sont situés près de la caisse de stérilisation et les cellules doivent donc être insensibles au rayonnement infra-rouge.

\section{B. - Description de l'appareillage de mesure}

\section{1) Cellules photoélectriques}

Les cellules choisies utilisent les propriétés photoconductrices du sulfure de cadmium activé par des cristaux d'argent ou de cuivre; elles présentent une sensibilité supérieure aux cellules à gaz ou au sélénium et sont peu sensibles au rayonnement infrarouge. La cellule est protégée par un petit boîtier métallique muni d'un dispositif éliminant les effets de la lumière ambiante ; elle est éclairée par un projecteur composé d'une ampoule de $6 \mathrm{~V}$ et d'une lentille de concentration du faisceau.

Le montage de l'ensemble cellule-projecteur est représenté sur les figures 3 et 4 . La cellule et le projecteur sont alignés suivant un axe vertical, la cellule étant à la partie inférieure; cette dispo- 
sition permet d'avoir toujours une couche de liquide entre' la cellule et le projecteur quel que soit le niveau du liquide.

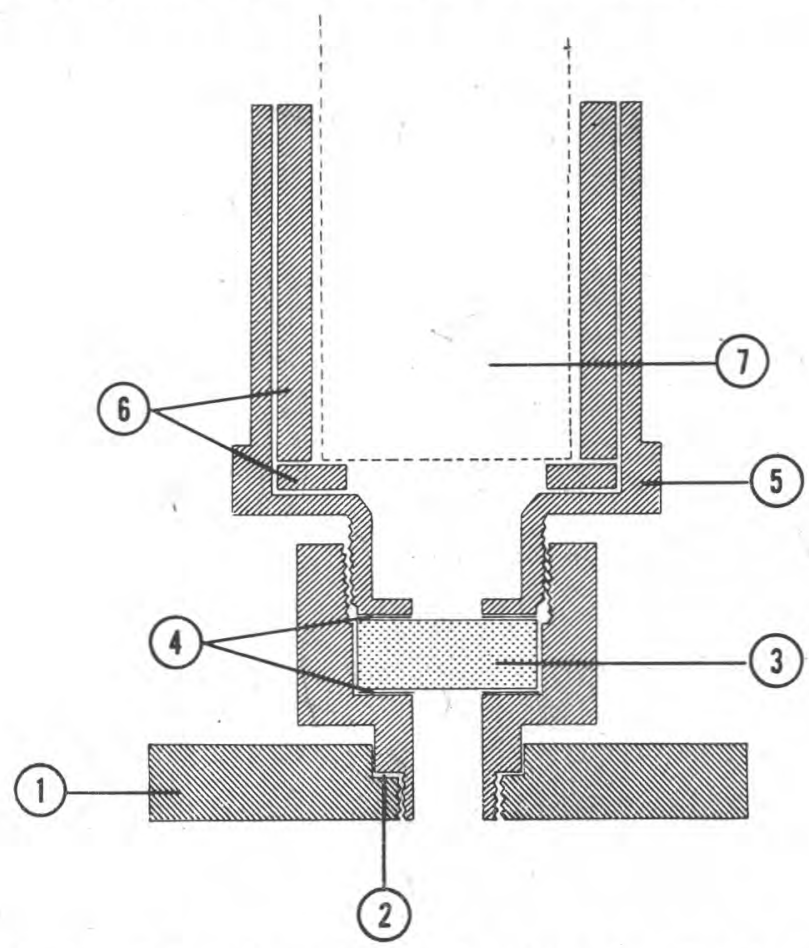

Fig. 3. - Détail du montage de la cellule photoélectrique.

(1) Tuyauterie; (2) Joint en teflon ; (3) Lame de verre à faces parallèles ; (4) Joint en klingérite; (5) Porte-cellule; (6) Gaine isolante ; (7) Cellule.

Fig. 4. - Vue du montage de la cellule photoélectrique au point de mesure $\mathbf{P}_{2}$.

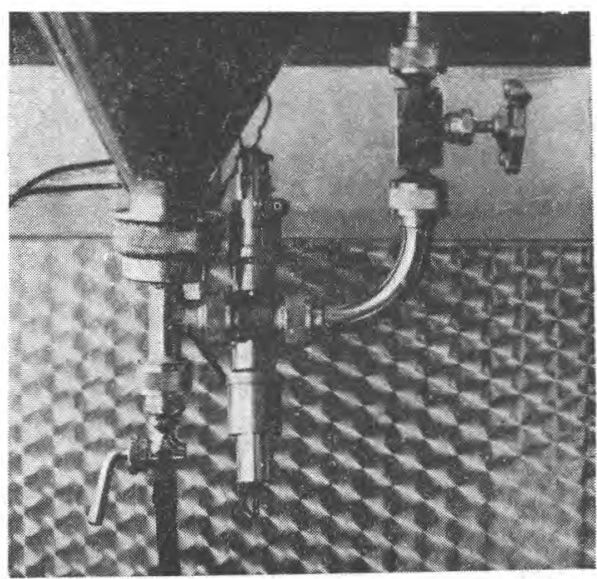




\section{2) Appareil enregistreur.}

Les variations de la quantité de lumière transmise, sont envoyées sous forme de variations d'intensité de courant électrique à un enregistreur galvanométrique à voies multiples (Technique Electronique, Paris, type EN 18). Les signaux sont enregistrés sur une bande de papier photographique de 160 millimètres de largeur dont la vitesse de déroulement a été fixée pour les essais à 4 centimètres par seconde. L'amplitude maximum des signaux sur le papier est de 3 centimètres.

3) Injection du colorant.

Le colorant est une solution aqueuse de bleu de méthylène à $0,1 \%$; cette concentration correspond à la concentration minimum entraînant une extinction totale de la cellule.

Un tuyau de caoutchouc gainé de toile, de 5 mètres de longueur et de 8 millimètres de diamètre intérieur, est rempli avec 150 millitres de la solution de bleu de méthylène; les deux extrémités du tuyau sont. alors fixées, l'une à la sortie d'un détendeur d'air comprimé, l'autre à la sortie d'un robinet $(R$, fig. 1) monté sur la tuyauterie du lait en aval de la pompe d'injection. Le stérilisateur étant en fonctionnement, deux opérateurs ouvrent simultanément, l'un le robinet du détendeur, l'autre le robinet $R$; après deux secondes, ils referment simultanément chacun leur robinet pendant qu'un troisième opérateur met en route l'enregistreur.

Entre deux mesures, le stérilisateur est soigneusement rincé avec 20 litres d'eau, pour éliminer toute trace de bleu de méthylène.

\section{C. - Résultats}

\section{1) Lecture des diagrammes.}

La figure 5 est la reproduction d'un diagramme d'enregistrement typique. La courbe de variation de quantité de lumière transmise peut être décomposée en trois sections.

Section $a b$ correspondant au passage de l'eau. Les perturbations tiennent aux faits suivants : au niveau de la cellule $P_{1}$, le tuyau n'est pas complètement rempli et les perturbations traduisent les variations de niveau du liquide puisque la cellule et le projecteur sont alignés suivant un axe vertical. Au niveau de la cellule $P_{2}$, passe un mélange de liquide et de vapeur; comme une bulle de vapeur diffracte les rayons lumineux, le passage de chaque bulle se traduit par une diminution de la quantité de lumière transmise.

Section bd correspondant au passage du front de la solution de bleu de méthylène. L'augmentation de l'opacité du liquide au niveau de la cellule se traduit par une diminution de la quantité de lumière transmise et aussi par une diminution de l'amplitude 


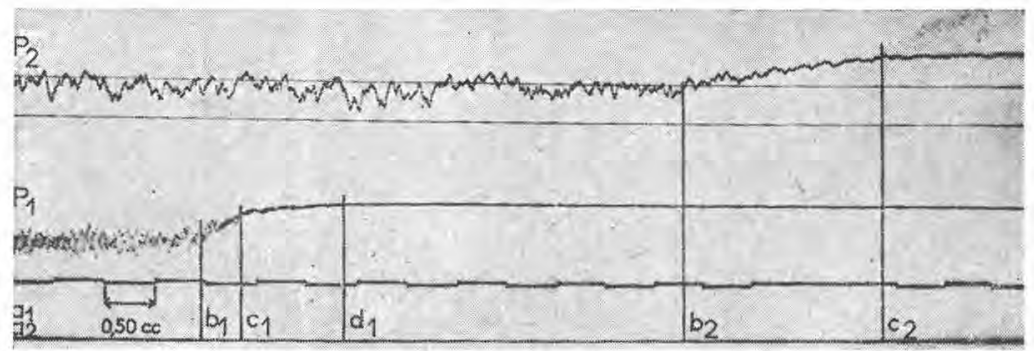

Fig. 5. - Diagramme d'enregistrement.

des oscillations de la courbe d'enregistrement puisque la sensibilité de la cellule diminue quand la quantité de lumière transmise diminue. On peut distinguer deux phases successives $b c$ et $c d$ : la première, $b c$, montre une diminution très rapide de la quantité de lumière transmise correspondant au passage des premières traces de bleu de méthylène; dans la deuxième, $c d$, on atteint lentement l'extinction complète de la cellule au moment où passe la solution pure de bleu de méthylène. Les durées respectives de ces deux phases sont : $b_{1} c_{1}=0,5$ et $c_{1} d_{1}=1$ seconde pour $\mathrm{P}_{1}, b_{2} c_{2}=2$ et $c_{2} d_{2}=3$ secondes pour la cellule $\mathrm{P}_{2}$. La durée de passage du front de la solution de bleu de méthylène, soit $b d$, est de 1,5 seconde au niveau de $\mathrm{P}_{1}$ et de 5 secondes au niveau de $\mathrm{P}_{2}$; la différence entre ces deux valeurs traduit les variations de vitesse à l'intérieur de la caisse de stérilisation. L'intervalle de temps qui sépare le début du passage de la solution de bleu de méthylène au niveau de chaque cellule correspond à la durée minimum de séjour dans la caisse de stérilisation, soit dans ce cas $b_{1} b_{2}=5$ secondes; l'intervalle de temps qui sépare l'extinction complète de chaque cellule correspond à la durée maximum du séjour dans la caisse de stérilisation, soit dans ce cas $d_{1} d_{2}=8,5$ secondes.

\section{2) Résultats proprement dits.}

Le tableau I résume l'ensemble des essais effectués; les valeurs de temps qui y figurent sont les durées minimum de séjour dans la caisse de stérilisation, l'écart entre les durées minimum et maximum restant toujours le même ( 3 à 4 secondes) sauf indication contraire.

Influence du débit. Pour les débits compris entre 60 et 120 litres/heure (essais 3 à 5), la durée du séjour est constante: sa valeur oscille entre 4,75 et 6 . Par contre, quand le débit est compris entre 15 et 30 litres/heures, la durée de séjour et l'intervalle de temps, qui sépare l'extinction complète de chaque cellule, augmentent. Par exemple, à $130^{\circ} \mathrm{C}$., pour un débit de 15 litres/heure, les durées minimum et maximum de séjour sont respectivement de 13,25 et 
22,5 secondes. Cette influence des débits trop faibles explique la valeur élevée (10 secondes) de la durée du séjour à $150^{\circ} \mathrm{C}$. : l'essai 10 a été effectué avec le débit maximum compatible à cette température de traitement avec la capacité de la chaudière, débit qui n'était que de 38 litres/heure.

Influence de la température de traitement. Quand le débit reste compris entre 60 et 120 litres/heure, la durée de séjour est pratiquement indépendante de la température.

Influence de l'ouverture de la vanne $V_{2}$ entre les deux caisses. A condition que l'ouverture soit suffisante pour ne pas provoquer d'accumulation du liquide dans la caisse de stérilisation, ce qui est le cas pour les essais du tableau, l'ouverture de la vanne $V_{2}$ n'a pas d'influence sur la durée de séjour dans la caisse de stérilisation.

Donc, en résumé, la durée du passage de la solution de bleu de méthylène est indépendante du débit, de la température de chauffage et de l'ouverture de la vanne entre les deux caisses, à condition que le débit de l'appareil soit d'au moins 60 litres/heure. Les durées minimum et maximum de chauffage sont respectivement de 5 à 6 et de 8 à 10 secondes.

\section{IV. - ACTION SPORICIDE DU STERILISATEUR}

Pour vérifier les résultats obtenus, on a étudié la destruction des spores d'une souche de Bacillus subtilis par chauffage dans le stérilisateur.

\section{1) Méthodes.}

Préparation des spores. - Lès spores utilisées sont celles d'une souche $\left(\mathrm{SJ}_{2}\right)$ de Bacillus subtilis isolée du lait bouilli par PLomMET [6]. Les spores sont préparées en boîte de Roux, suivant la technique de Plommet [6], lavées par centrifugation répétée à $4^{\circ} \mathrm{C}$. et conservées en tampon phosphate $\mathrm{M} / 15$ à $p \mathrm{H} 7,0$, à $4^{\circ} \mathrm{C}$.

La numération de la suspension ainsi obtenue est effectuée avec la cellule de Petroff-Hausser.

Thermorésistance au laboratoire. - Des ampoules de verre ordinaire, de 10 millimètres de diamètre intérieur et contenant $10_{6}$ spores, dans 1 millilitre de tampon phosphate $\mathrm{M} / 15 p \mathrm{H}$ 7,0, sont chauffées dans un bain-marie de glycérine dont la température est maintenue constante à $\pm 0,2^{\circ} \mathrm{C}$. près. A intervalles réguliers des ampoules sont sorties du bain-marie et immergées immédiatement dans l'eau glacée.

La numération des spores après traitement est effectuée en milieu gélosé peptoné, après incubation de 6 jours à $30^{\circ} \mathrm{C}$.

Thermorésistance dans le stérilisateur. - Les conditions de chauffage (milieu et concentration en spores) et de numération des spores survivantes sont les mêmes que précédemment. 


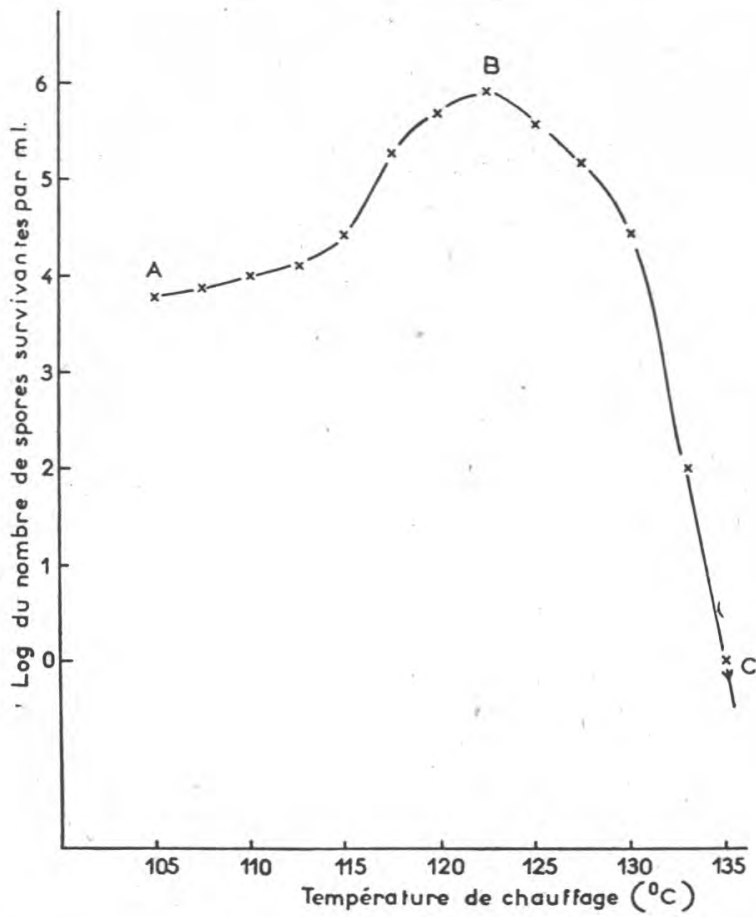

Fig. 6. - Destruction des spores de Bacillus subtilis $\mathrm{SJ}_{2}$ dans le stérilisateur. $\mathrm{AB}$ : courbe d'activation. $\mathrm{BC}$ : courbe de destruction.

\section{2) Résultats.}

Les résultats concernànt la destruction des spores de Bacillus subtilis dans le stérilisateur sont représentés dans la figure 6 . La destruction des spores ne commence qu'à $125^{\circ} \mathrm{C}$.; à $135^{\circ} \mathrm{C}$. le nombre de spores survivantes est inférieur à $1 / \mathrm{ml}$. La destruction (portion $\mathrm{BC}$ de la courbe) est précédée d'une activation particulièrement nette (portion $A B$ de la courbe) ; le phénomène d'activation présenté par cette souche a déjà été mis en évidence par Plommet [6] et est actuellement à l'étude [4] [5].

La figure 7 représente la courbe des temps de destruction thermique (courbe TDT), qui donne pour chaque température de chauffage la durée minimum de chauffage pour abaisser la concentration en spores viables de $10_{6}$ à moins de $1 / \mathrm{ml}$. de tampon. La courbe a été construite suivant les critères de Townsknd et al. [7], d'après les résultats obtenus au laboratoire, pour des températures de chauffage allant de 105 à $120^{\circ} \mathrm{C}$. Puisque cette courbe est linéaire, il est facile de l'extrapoler graphiquement pour des températures de chauffage supérieures à $120^{\circ} \mathrm{C}$. On peut ainsi calculer que la 


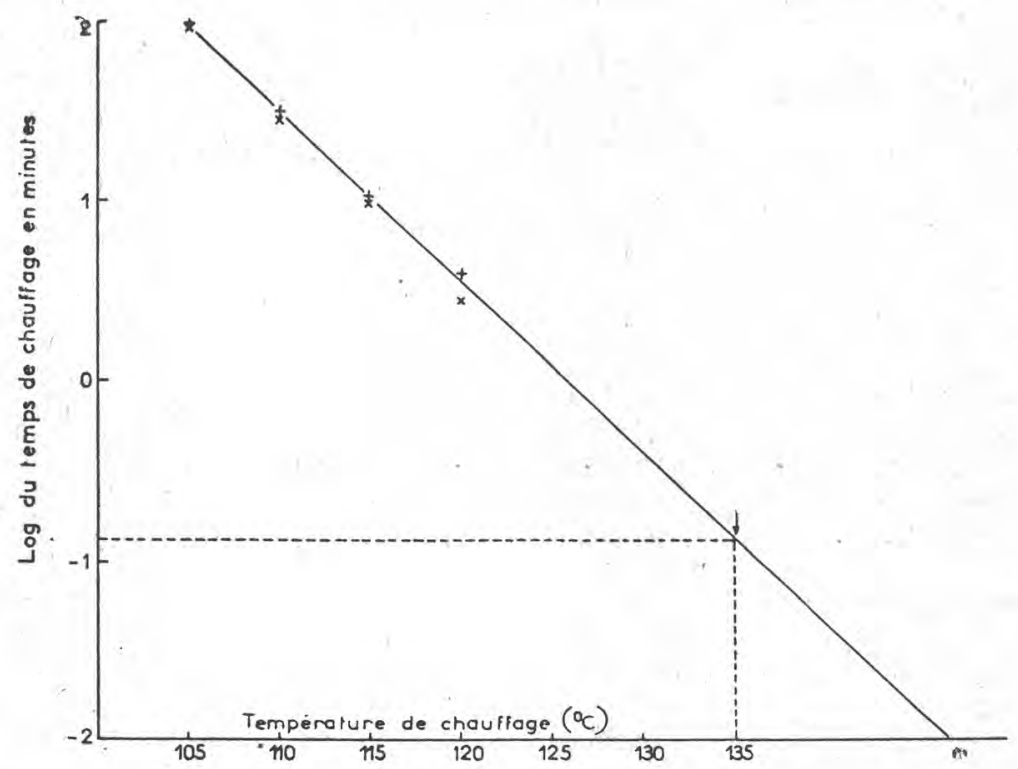

Fig. 7. - Courbe des temps de destruction thermique des spores de Bacillus subtilis en tampon phosphate. $\times$ Point maximum de survie; + Point minimum de destruction.

destruction de $10_{6}$ spores dans 1 millilitre de tampon phosphate, exige à $135^{\circ}$ C., un temps de chauffage d'au moins 8 secondes (0,13 minute). Cette valeur "théorique» est en bon accord avec la valeur trouvée par mesure de la vitesse de passage de la solution de bleu de méthylène.

\section{RÉSUMÉ}

Il est possiblę de mesurer la durée du chauffage du lait dans un préstérilisateur (appareil de stérilisation à très haute température) en observant le passage d'une solution de bleu de méthylène à l'aide de cellules photoélectriques. Cette technique est utilisable même quand la tuyauterie au niveau de la cellule n'est pas complètement pleine; elle convient très bien aux régimes turbulents et aux mélanges liquide-vapeur à condition que les bulles de vapeur soient en nombre faible.

Dans le cas d'un stérilisateur de laboratoire construit sur le principe du préstérilisateur Laguilharre (chauffage par pulvérisation du lait dans une atmosphère de vapeur sous pression), les durées minimum et maximum de chauffage sont respectivement de 5 à 6 et de 8 à 10 secondes; ces durées sont indépendantes de 
la température de chauffage et du débit dans les limites de fonctionnement normal de l'appareil.

L'effet sporicide du préstérilisateur a été mesuré avec une souche de Bacillus subtilis: un chauffage à $135^{\circ} \mathrm{C}$. permet un taux de destruction de $99,9999 \%$. La valeur calculée du temps de chauffage d'après la thermorésistance des spores concorde bien avec celle trouvée par les mesures physiques.

(Nous exprimons notre reconnaissance à M. Mocquot qui par sez conseils, nous a guidé tout au long de la réalisation de ce travail).

\section{BIBLIOGRAPHIE}

[1] H. Burton. An analysis of the performance of an ultra-high-temperature milk sterilizing plant. I. Introduction and physical measurements. Journal Dairy Res., 25, 75 à 84. 1958.

[2] J. G. Davis. Observations on the control of the H.T.S.T. process. Dairy Ind., 18, 682 à 689. 1953.

[3] J. Hermer. Une méthode rapide de détection de la non-stérilité éventuelle d'échantillons de lait chauffé au-dessus de $100^{\circ} \mathrm{C}$. Ann. Inst. Nat. Rech. Agron., Série E (Ann. Technol. Agric.), 6, 377 à 390. 1957.

[4] J. Hermier. L'activation par la chaleur de la germination des spores de Bacillus subtilis. C. R. Acad. Sci,, 246, 3298 à 3300.1958.

[5] J. Hermier. L'influence d'un préchauffage en présence de glucides sur la germination des spores de Bacillus subtilis. C. R. Acad. Sci., 246, 3388 et 3389.1958.

[6] M. Plommet. L'ébullition domestique du lait. Son action sur la flore microbienne et les constituants du lait. Ann. Inst. Nat. Rech. Agron., série E (Ann. Technol. Agric.), 5, 493 à 519. 1956.

[7] C. T. Townsend; J. R. Esty et F. C. BAselt. Heat resistance studies on spores of putrefactive anaerobes in relation to determination of safe processes for canned foods. Food. Res., 3, 323 à 346.1938.

\section{DOSAGE NÉPHÉLO-COLORIMÉTRIQUE DU LACTOSE (1)}

par

J. C. GODFRAIN, P. BERTRAND et Mlle Léonce LiANDIER

Poursuivant nos recherches sur l'application à l'analyse chimique des substances tensio-actives [1] [2] [3], il nous a paru intéressant d'essayer d'appliquer notre technique de dosage des sucres réducteurs [1] en présence de Teepol 710 (Le Teepol 710 contient $40 \%$ d'alkyl-sulfate secondaire à 12 atomes de carbone environ), au lactose dans le lait.

(1) Bull. Acad. Vét., 1958, 31, no 6, 249. 